\title{
Image Processing with ImageJ
}

by Dr. Michael D. Abràmoff, University of lowa Hospitals and Clinics; Dr. Paulo J. Magalhães, University of Padua; and Dr. Sunanda J. Ram, Louisiana State University Health Sciences Center

As the popularity of the ImageJ open-source,
Java-based imaging program grows, its capabilities increase,
too. It is now being used for imaging applications
ranging from skin analysis to neuroscience.

T he advances of the medical and biological sciences over recent years, and the growing importance of determining the relationships between structure and function, have made imaging an increasingly important discipline. The ubiquitousness of digital technology from banal digital cameras to highly specific micro-CT scanners - has made images an essential part of a number of re- search areas, from nanotechnology to astronomy.

It is common practice for manufacturers of image acquisition devices to include dedicated image processing software, but these programs are usually not very flexible and/or do not allow more complex image manipulations. Image processing programs also are available by themselves.

ImageJ holds a unique position because

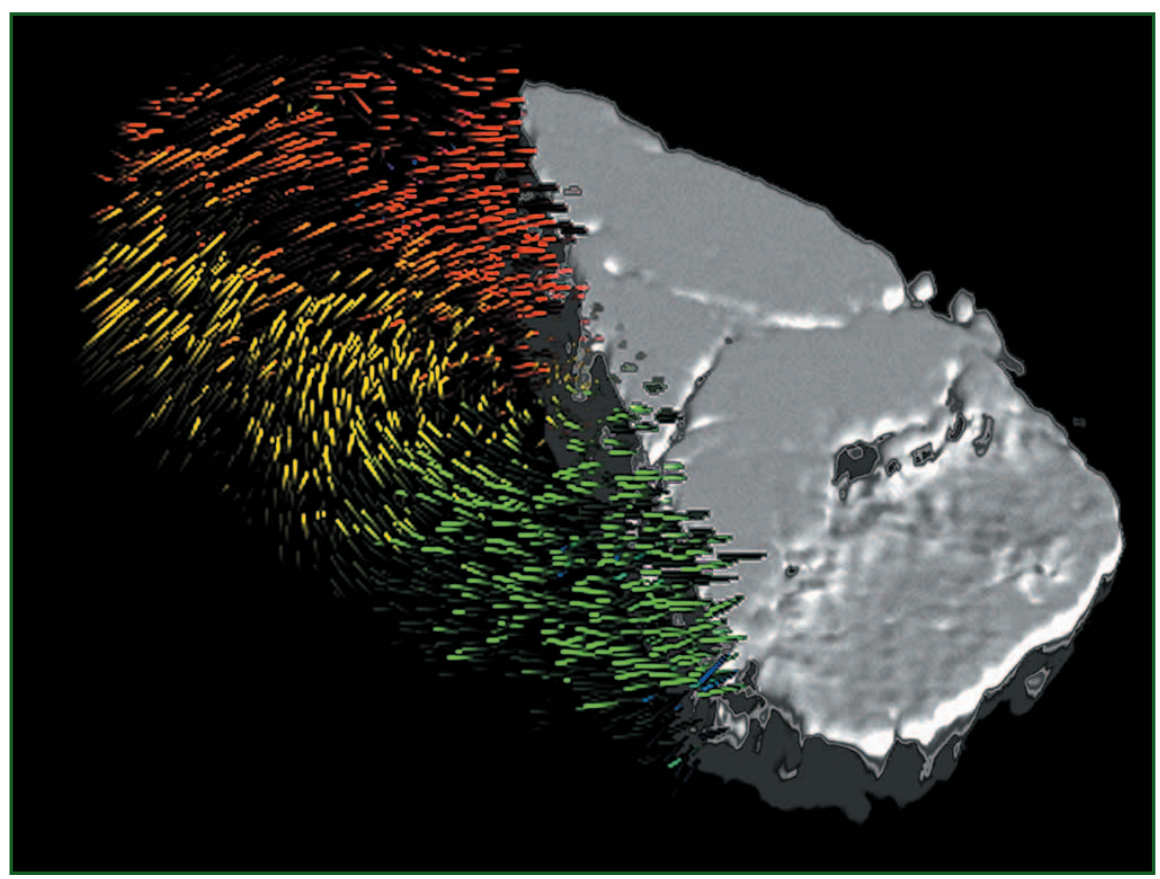

it not only is in the public domain (meaning that its source code is openly available and its use is license-free), but also runs on any operating system. It is attractive because it is easy to use, can perform a full set of imaging manipulations and has a huge and knowledgeable user community.

The program is the brainchild of Wayne Rasband of the Research Services Branch, National Institute of Mental Health, in Bethesda, Md. It is called ImageJ because it is written in the Java language. Its first release, version 0.50, was Sept. 23, 1997, and it is now in version 1.31, released in February.

According to the institute, it has been downloaded from its Web site tens of

Figure 1. A scintillation rendering of the 3-D motion field of a fat-muscle phantom was combined with a surface rendering to show motion and anatomy of the phantom. The motion data were obtained as 1.5-T MRI gradient echo T1 weighted image sequences, while the phantom was rotated in place at $5^{\circ}$ per frame. The motion field is too dense for motion and surface to be rendered in the same place. The axis of rotation was oriented perpendicular to the surface of the phantom. The hue of the color codes for direction, and the saturation of the color codes for motion magnitude. Obtained with ImageJ running VolumeJ for volume rendering and FlowJ for 3-D optical flow estimation. Provided by Michael Abràmoff.

Reprinted from the July 2004 issue of Biophotonics International @ Laurin Publishing Co. Inc. 


\section{IMAGING SOFTWARE}

thousands of times, with the current rate being about 24,000 downloads per month. Rasband said that, after working for 10 years on NIH Image, ImageJ's precursor, he didn't see a bright future for it, even though Scion Corp. of Frederick, Md., had ported it from a Macintosh-only version to the PC/Windows platform. However, the Scion version wasn't open source, and he is a strong proponent of open source and platform independence. So he began writing Image in early 1997 after he had become intrigued by the new Java programming language.

\section{Imaging capabilities}

Image J can read most of the common and important formats used in the field of biomedical imaging (see table). If a

file format is not currently supported, someone from the international user/ developer community usually develops support within days. In addition, ImageJ can be used to acquire images directly from scanners, cameras and other video sources, including cameras that are compatible with TWAIN and FireWire, and frame grabber boards from Cooke, National Instruments and PixelSmart.

The program supports all common image manipulations, including reading and writing of image files, and operations on individual pixels, image regions, whole images and volumes. Volumes, called stacks in ImageJ, are ordered sequences of images that can be operated upon as a whole. It can perform basic operations such as convolution, edge detection, Fourier transform, histogram and particle analyses (including sophisticated statistical processing of groups of particles), editing and color manipulation; and more sophisticated operations such as dilation, erosion and closing of structures, and mathematical operations on sets of images, such as multiplication, exclusive or, and division. In addition, visualization operations, including color space conversions - for example, converting from RGB to Hue Saturation Intensity color space, two- and three-dimensional plotting - and surface and volume rendering, are supported. It also offers core support for analyzing electrophoretic gels.

\section{Cross-platform}

One of the strong points of ImageJ is its ability to run on different platforms. Statistics covering the last three months, with more than 80,000 downloads, indicate that it is being used mostly with Microsoft operating systems (80 percent), followed by Macintosh platforms (16 percent) and Linux (4 percent). Although these numbers are estimates and can be misleading because someone could download it to one platform and use it on another, it is interesting to note the relatively high number of Macintosh users (the worldwide market share was less than 2 percent in 2003, according to market research company IDC), supporting the long-held view that this type of computer attracts large sections of academia.
Obviously, with a free program, there is no telephone hot line for support. However, a large user base communicates through a mailing list. This way, any user is free to ask questions, put forward suggestions or ideas for new imaging functions and publish solutions. At the time of this writing, this community consisted of more than 1000 users/ scientists (the majority) and users/developers (in smaller numbers, but of fundamental importance).

Indeed, the know-how of the community is remarkably high because many members possess a detailed knowledge of the software and the imaging problems that the program can address efficiently. A request on the mailing list is usually all it takes for someone, somewhere in the world, to provide the required help.

For example, Dr. Rex Couture of the department of radiology at Washington University School of Medicine in St. Louis ran into a problem with reading large micro-CT images. These files were about 5 GB, but ImageJ couldn't read beyond the 4-GB limit at that time. "I posted a query about this," Couture said. "Within an hour or two, someone working through the night in Europe had found the problem. By 9:30 the next morning, I had a solution to try from Wayne. Since he didn't have an image file that large, he couldn't test it, and it didn't quite work. Twenty-four hours later he had created a test image, and I had a fully debugged new version that solved the problem." Shortly thereafter, someone in the discussion group also posted a way to open large tiff files.

There has always been a somewhat terse usage instruction on the ImageJ Web site, but getting advice on a specific function required taking the quite intimidating step of asking the mailing list. Now one has the option of using a manual recently produced by Tony Collins at the Wright 
Figure 3. This simple ImageJ macro acquires an image every 10 seconds and stores it in sequence.

Cell Imaging Facility at Toronto Western Research Institute. This free, very thorough manual has many examples and illustrations. It emphasizes microscopy and accompanies a collection of microscopyrelated plug-ins. Although many opensource programs lack an extensive user manual, even novices can find most of the information for their imaging needs in this manual.

\section{Extensions: Macros and plug-ins}

The program is virtually limitless because of the availability of user-written macros and plug-ins.

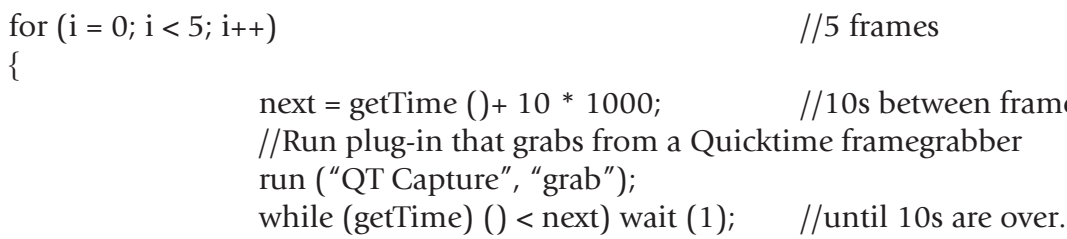

Macros are meant to make it easier to automate oft-repeated tasks, which would be tedious to implement manually. ImageJ has an easy-to-use macro-language that means that knowledge of Java isn't required for writing simple scripts. For example, a macro can be written that acquires an image every 10 seconds and stores it in a sequence (Figure 3 ).

Plug-ins are external programs, mostly written in the Java language, that offer image processing capabilities that do not

\begin{tabular}{|c|c|}
\hline \multicolumn{2}{|c|}{$\begin{array}{l}\text { TABLE } \\
\text { Image formats supported by ImageJ as of June } 2004\end{array}$} \\
\hline Format & Read and write \\
\hline Analyze (Mayo Clinic's format) & (plug-in) \\
\hline AVI uncompressed movies & $\sqrt{2}$ \\
\hline Bio-Rad Z-series & (plug-in) \\
\hline BMP & $\checkmark$ \\
\hline DICOM (uncompressed) read & $\checkmark$ \\
\hline DICOM (uncompressed) write & (plug-in) \\
\hline DM3 (Gatan Corp. transmission electron microscopy format) & (plug-in) \\
\hline $\begin{array}{l}\text { FDF, VFF (Varian MRI imaging system/EVS } 900 \text { micro-CT system } \\
\text { formats) }\end{array}$ & (plug-in) \\
\hline FITS (NASA format) & read \\
\hline GIF (including animated) & $\checkmark$ \\
\hline ICO, CUR (MS Windows) & (plug-in) \\
\hline IPLab (Scanalytics Corp. microscopy format) & (plug-in) \\
\hline Jpeg & $\checkmark$ \\
\hline Jpeg EXIF digital camera header & (plug-in) \\
\hline Leica SP multichannel & (plug-in) \\
\hline LSM (Zeiss LSM 510 confocal microscopes) & (plug-in) \\
\hline PCX (Zsoft Corp.) & (plug-in) \\
\hline PDS (Planetary Data System) & (plug-in) \\
\hline PGM (P2 and P5) & read \\
\hline PICT (Apple Mac) & (plug-in) \\
\hline PNG & $\sqrt{2}$ \\
\hline PSD (Adobe Photoshop) & (plug-in) \\
\hline QuickTime movies & $\sqrt{2}$ \\
\hline $\begin{array}{l}\text { Raw (any uncompressed file format not directly supported, } \\
8-, 16-, 24 \text {-bit } R G B, 32-\text { bit, small- and big-endian) }\end{array}$ & $\checkmark$ \\
\hline SIF (Andor Technology spectroscopy format) & (plug-in) \\
\hline SPE format (Roper Scientific cameras) & (plug-in) \\
\hline Sunraster & (plug-in) \\
\hline Targa & (plug-in) \\
\hline Text images (tab or comma separated) & $\checkmark$ \\
\hline $\begin{array}{l}\text { Tiff uncompressed and zip compressed, both as } \\
1-, 8-, 12-, 16-\text { bit unsigned, 32-bit (real) } \\
\text { or RGB color, single and multiple images (stacks) }\end{array}$ & $\checkmark$ \\
\hline XBM, XPM (X-Windows formats) & (plug-in) \\
\hline XLS (Microsoft Excel) & (plug-in) \\
\hline
\end{tabular}

exist in core capabilities of ImageJ. Once implemented, they cannot be distinguished from the program itself. A small cottage industry has sprouted from users/ developers who are designing plug-ins for their own use and sharing them with all users. Plug-ins have brought ImageJ from an image processing program to a framework that scientists can use to develop their own imaging solutions.

Plug-ins range from very small and straightforward, such as the Grid plug-in - which simply draws a grid on an image - to complex, practically stand-alone image programs, such as the dendritetracing tool Neuron $J^{1}$ or the surface and volume-rendering plug-in VolumeJ. ${ }^{2}$ In fact, the standard imaging capabilities that ImageJ comes with are implemented as plug-ins as well. Developing new plugins requires knowing Java language and is thus not for everyone.

However, after using ImageJ for a while, users often need a specific solution for their problem that goes beyond its core capabilities. The Medientechnik und design group in Austria has written a tutorial specifically as an introduction to writing plug-ins for ImageJ.

Most plug-ins that are judged by their developers to be of general use can be published on the ImageJ Web site, which now holds more than 150 plug-ins, written by 98 developers. Some developers may instead make their plug-ins and macros available through personal Web sites; some of these are listed on the ImageJ site

A third way of extending ImageJ, which is used by only a small number of more technically advanced developers, is to use its imaging capabilities and plug-ins from their own programs, so that, in technical terms, they are using it as a library of imaging methods. This is called ImageJ's application programmer's interface (API), which has been extensively documented so that it is clear how to use and carry out these methods. This way, several online image database servers have been

\section{Imaging library}


Figure 4. The main window in the back shows a fluorescence microscopy image (compiled from several scans) of a neuronal cell and its outgrowth, together with the primary (red), secondary (blue) and tertiary (magenta) tracings. Superimposed are the ImageJ window (top left) with the NeuronJ toolbar (replacing the ImageJ toolbar during operation) and two NeuronJ result windows (bottom right), giving the length of individual tracings and statistics on selections of tracings. Courtesy of Erik Meijering, Erasmus MC-University Medical Center Rotterdam.

developed, including one for ophthalmologic telediagnosis.

The applications to which ImageJ has been applied are astounding. Space limitations dictate that only a few salient examples can be given here: Paulo Magalhães and co-workers in the department of biomedical sciences at CNR Institute of Neuroscience, University of Padua, Italy, are using it to study the dynamics of intracellular calcium, ${ }^{3,4}$ while researchers at the Laboratory for Cellular Neurobiology of the Swiss Federal Institute of Technology in Lausanne and the Biomedical Imaging Group at Erasmus MC-University Medical Center Rotterdam in the Netherlands, are using the NeuronJ plug-in, for the automated quantitative analysis of neurons and dendrites (Figure 4). ${ }^{1}$ The NeuronJ plug-in is based on recently developed and validated algorithms for detecting and linking elongated image structures specifically for this purpose.

Andrea Mothe and co-workers at the department of zoology at the University of Toronto at Scarborough, in collaboration with Collins from Toronto Western Research Institute, use ImageJ and the VolumeJ plug-in for the 3-D reconstruction of the differential localization of nerve cell gene expression (Figure 5).

Procter \& Gamble in Cincinnati is using ImageJ as a framework for Visia, a system that carries out complexion analysis by comparing skin texture analysis of a subject's facial skin against the texture analyses of other subjects stored in a database. The program uses the ImageJ application programmers interface (API) as a library (Figure 6)

The Biomedical Imaging Group of the Swiss Federal Institute of Technology is
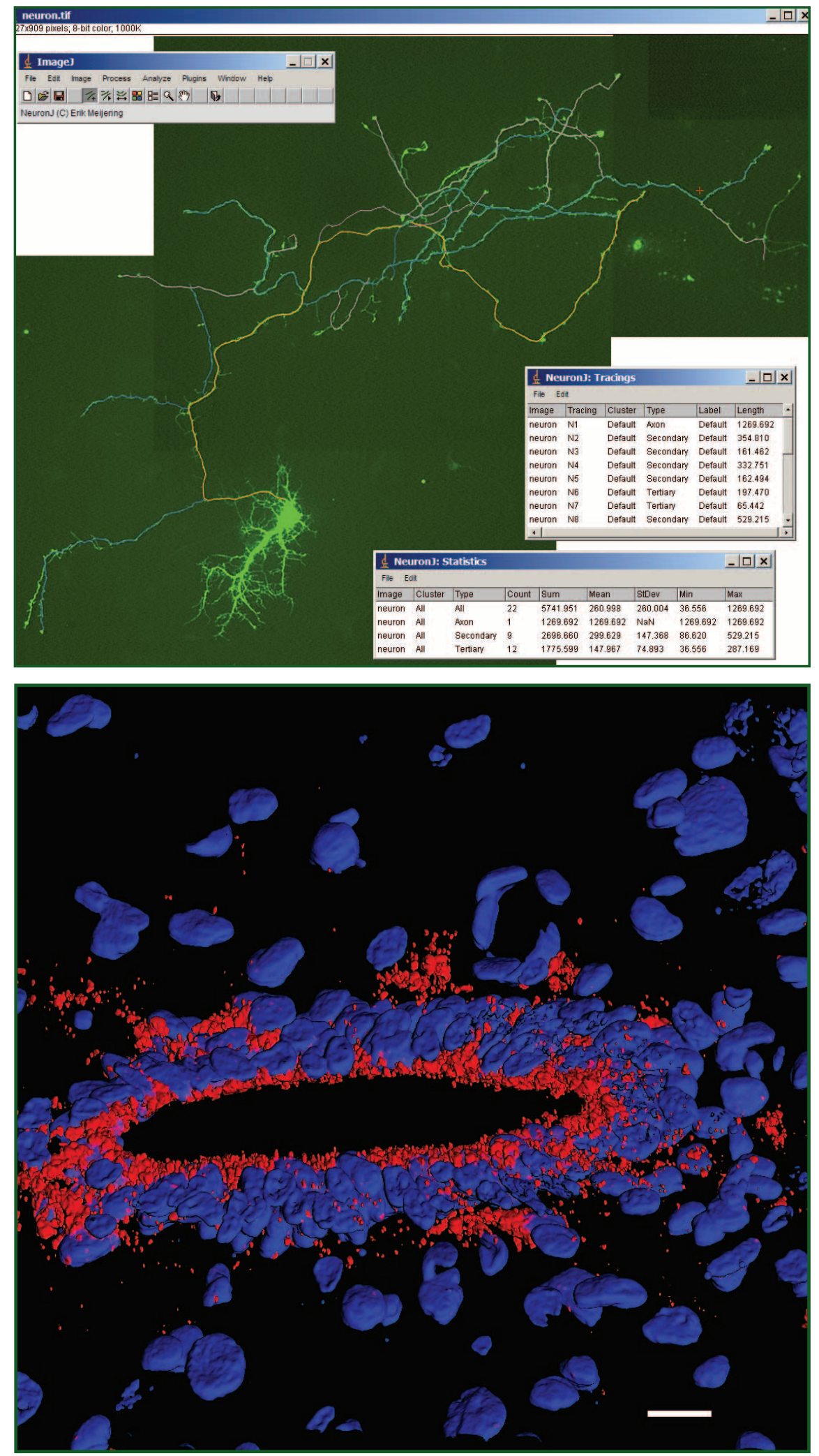

Figure 5. This 3-D reconstruction of DAPI-stained (blue) nuclei in a rat spinal cord section after intraventricular Dil injection results in red-staining of cells in the ependymal cell layer of the central cord. Scale bar $=10 \mu \mathrm{m}$. Image processed with ImageJ running VolumeJ and provided by Andrea Mothe and Tony Collins, Toronto Western Research Institute. 
using the program to teach image processing to engineering students, ${ }^{5,6}$ and the Center for Image Processing in Education

of Tucson, Ariz., is using it for teaching basic imaging to high school students. ${ }^{7}$

Dr. Michael D. Abràmoff and co-work-

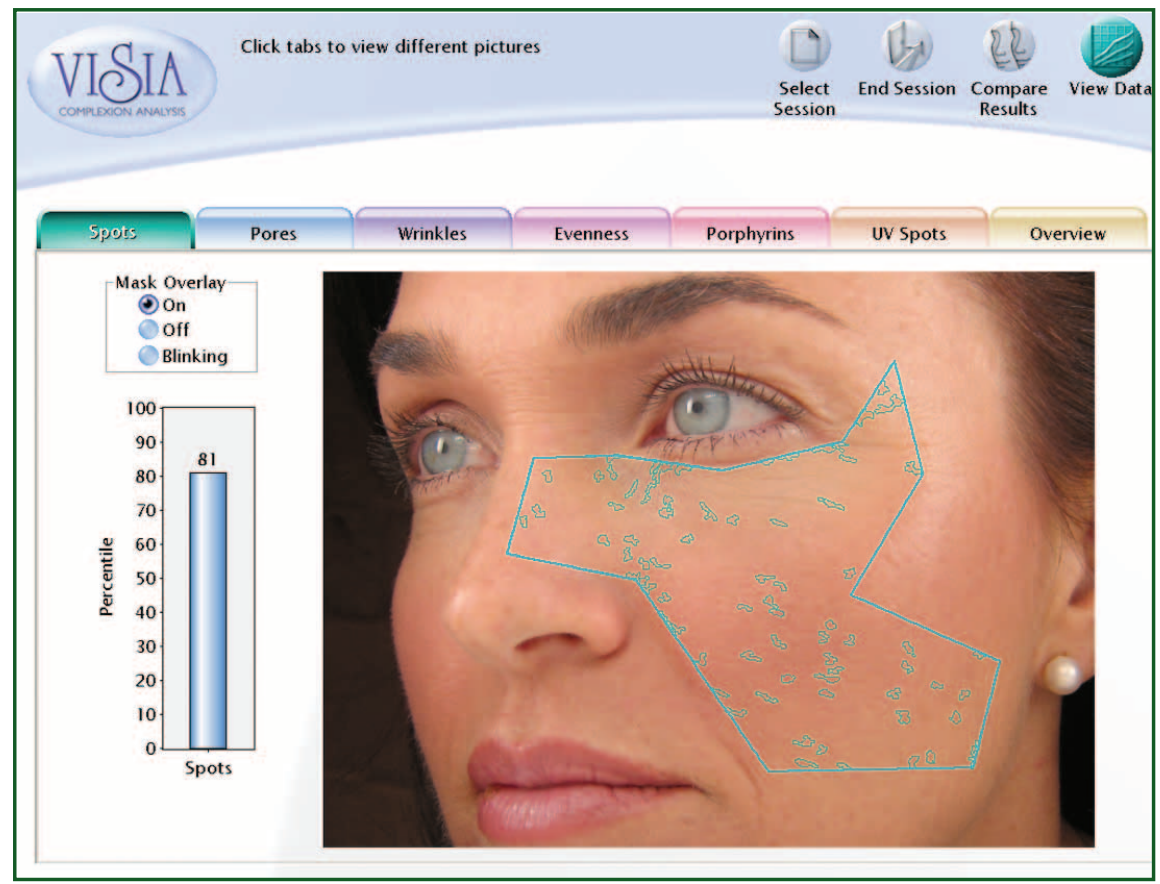

Figure 6. The Visia complexion analysis system uses ImageJ to analyze how the client compares with a database of women in her age and skin-type groups for the given categories - in this example, spots. It includes scores that indicate the percentage of women who scored below the client's evaluation for the category. Provided by Procter \& Gamble.

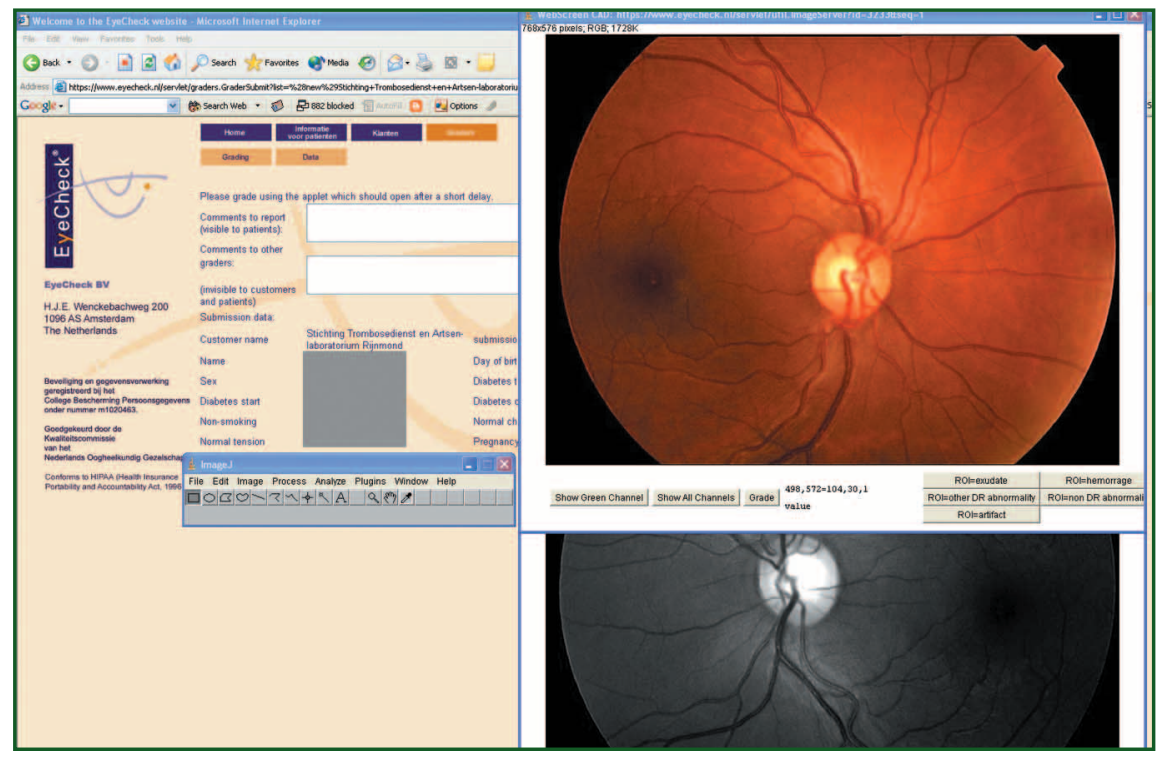

Figure 7. The EyeCheck Web site provides online diabetic retinopathy screening from retinal color photographs. The left area of the image shows the Web page with patient information. When this page is accessed, an ImageJ applet automatically displays the retinal photographs (in this case, of a normal subject), at right. This allows for user interaction so that, for example, the intensity image can be displayed, as in the bottom part, or specific abnormalities can be marked. Courtesy of EyeCheck. ers at the departments of ophthalmology and radiology at University Hospital Utrecht in the Netherlands, have used Image and the FlowJ and VolumeJ plugins for the differentiation of orbital tumors and for measuring the motion of soft tissues in patients. ${ }^{2,8}$

Abràmoff also worked with other clinicians to develop the EyeCheck Web site, an online diabetic retinopathy screening project in the Netherlands. It uses ImageJ's capabilities for storing and displaying retinal images in a telediagnosis environment. The program can accept and store a great variety of image formats, which can then be used for manual grading by ophthalmologists or for semiautomated or automated detection of diabetic retinopathy (Figure 7)

Finally, Dr. Peter Hurd at the department of psychology, University of Alberta in Edmonton, Canada, is using ImageJ to determine the second to fourth digit ratio (index to ring finger) in Anolis carolinensis, or anole lizard, which is thought to reflect the relative concentration of (or sensitivity to) androgens during development (Figure 8).

The program illustrates that imaging is on the boundary between being a field of science and a field of engineering. The solutions being proposed by and implemented in conjunction with users and developers are sometimes engineering solutions (in that they are derived straight

Figure 8. Imagel is being used to examine the second to fourth digit ratio (index to ring finger) in the anole lizard, which is thought to reflect the relative concentration of or sensitivity to androgens during development. Provided by Peter Hurd, University of Alberta.

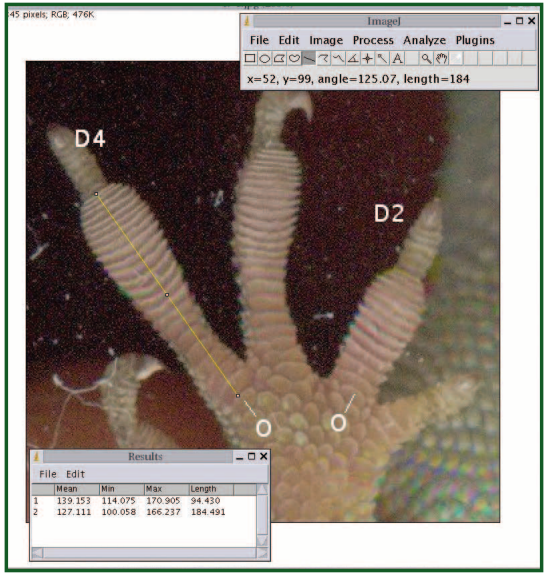




Figure 9. The SmartRoot system, an
interactive plant root image analysis
plug-in, was developed by Xavier
Draye and co-workers at the Unité
d'Ecologie des Grandes Cultures,
Université Catholique de Louvain,
Louvain-la-Neuve, Belgium. The tool
helps the user track root objects,
displays them in different "layers,"
saves them in Extend Markup
Language documents and sends
measurements to any Standardized
Query Language-compliant
databases. Courtesy of Xavier Draye.

from textbook material or a publication) and sometimes scientific solutions (when necessity dictates a scientifically new approach to solve a real-world problem).

A critical and pragmatic reader may expect some drawbacks to ImageJ, and there are some issues. The program requires minimal computer knowledge for installation and first steps, while commercial vendors may offer on-site installation and training. Also, because of the continuous state of development, bugs and "undocumented features" can creep into the distributed version. This can be a problem for the unaware researcher who compares data acquired with the old and new versions. Users usually spot these problems, and corrected versions of the program are made available immediately.

There is a misconception that an imaging program written in Java cannot be fast, and this may divert some potential users from ImageJ. Abràmoff used to share this worry and tested its validity by rewriting some of his convolution routines (which he had originally implemented in Java) in $\mathrm{C}++$ and calling these routines

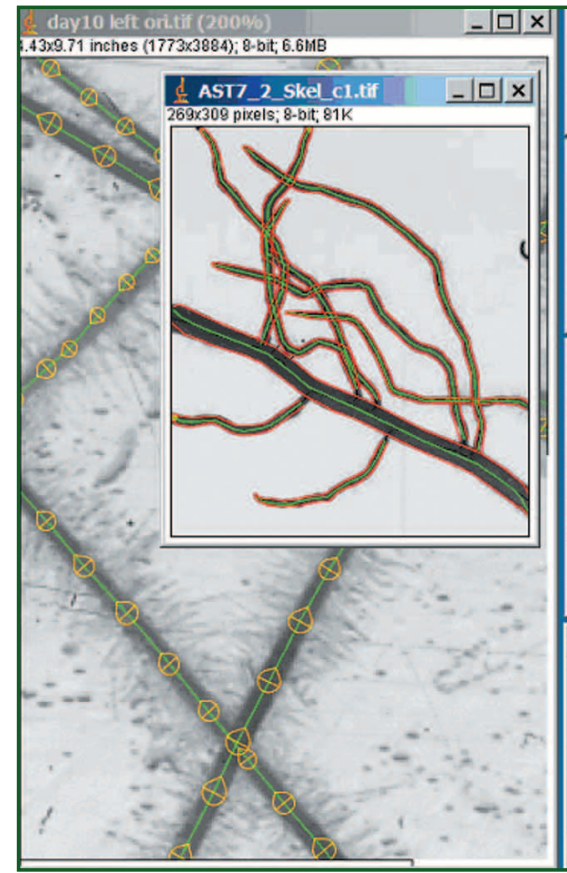

from a plug-in using the ImageJ Java Native Interface API. Although there was a decrease in processing time of about 30 percent, this did not weigh up to the increased development time for the routines.

Furthermore, there is no inherent reason why an algorithm coded in $\mathrm{C}++$ will run significantly faster than the same algorithm coded in Java. The execution speed depends on how good a compiler is at optimizing the generated code. Many people will even argue that the compilers that translate Java byte code into machine code can do a better job of optimization because more information is available to them about the program being compiled and about the machine that it is running on. So the reason why some commercial image processing pro-

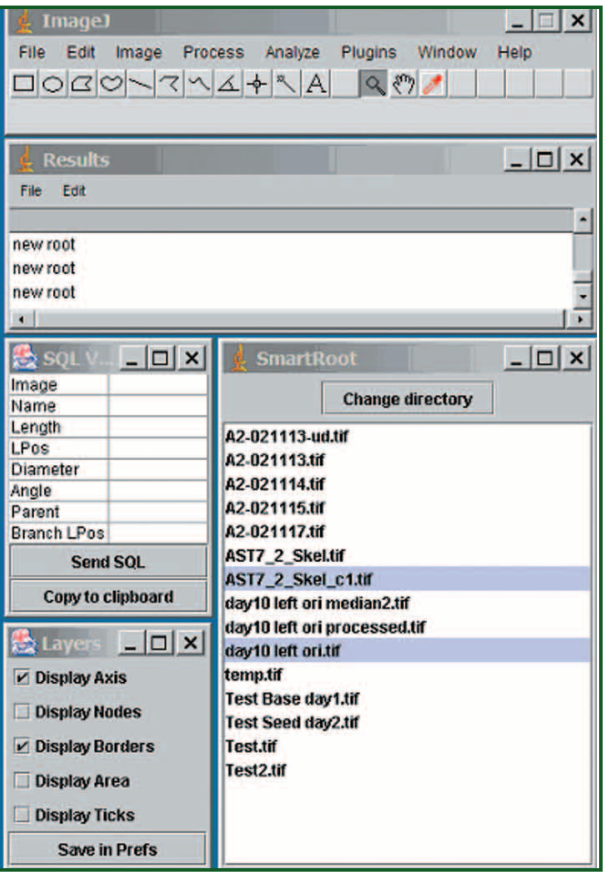

grams are appreciably faster than ImageJ at convolutions or similar processor-intense operations is related to increased sophistication of their algorithms. The open nature of ImageJ, however, may enable end-users with inspiring insights to develop better and more sophisticated algorithms, without the constraints of proprietary code.

In summary, ImageJ has attracted a varied and dedicated group of users because it is free and expandable, and can operate on any platform. It is especially remarkable how robustly the framework, designed six years ago, has withstood the test of time. Though it is difficult to predict where the program will be five years from now, the evolution will probably be a very interesting and rewarding experience for both users and developers.

\section{Meet the authors}

Dr. Michael D. Abràmoff, ophthalmologist and image science engineer, is at the department of ophthalmology and visual sciences, University of Iowa Hospitals and Clinics, Iowa City, Iowa. He is the author of VolumeJ and many other popular plug-ins; e-mail: michael abramoff@uiowa.edu.

Paulo J. Magalhães is at the department of biomedical sciences, University of Padua in Italy. He has been a contributor of the popular Ratio Plus plug-in.

Sunanda J. Ram is assistant professor of medicine at the Louisiana State University Health Sciences Center in Shreveport.

\section{Acknowledgment}

The authors wish to thank Wayne Rasband, without whom neither ImageJ nor its community would have existed.

Please send requests for additional copies of this article to biophotonics@laurin.com.

\section{References}

1. E. Meijering et al (April 2004). Cytometry 58A, pp. 167-176.

2. M.D. Abràmoff et al (February 2002). Invest Ophthalmol. Vis. Sci. 43, pp. 300-307.

3. M.F. Abad et al (March 19, 2004). J Biol
Chem 279, pp. 11521-9.

4. L. Filippin et al (Oct. 3, 2003). J Biol Chem 278, pp. 39224-34.

5. D. Sage and M. Unser (2001). Proc. of the 2001 IEEE International Conference on Image Processing, Thessaloniki, Greece 3, pp. 298301.

6. D. Sage and M. Unser (2004). IEEE Signal Processing Magazine 20, pp. 43-52.

7. J.V. Ekstrom (2000). The Science Teacher 67, pp. 53-55.

8. M.D. Abràmoff and M.A. Viergever (2002). IEEE Trans. Med. Imaging 21, pp. 296-304. 


\section{Web Resources}

- rsb.info.nih.gov/ij - ImageJ and most of its plug-ins and macros are available for download.

- www.uhnres.utoronto.ca/wcif/download.html - ImageJ manual by Tony Collins.

- mtd.fh-hagenberg.at/depot/imaging/imagej - The Medientechnik und design group tutorial on writing plug-ins.

- imagescience.bigr.nl/meijering/software/neuronj - NeuronJ is available for download.

- bij.isi.uu.nl - Web site for VolumeJ and many other biomedical imaging plug-ins.

\section{Publications in which ImageJ was used, listed by application}

\section{Function and dynamics of cells and organelles}

- M. Ghosh et al (April 30, 2004). Cofilin promotes actin polymerization and defines the direction of cell motility. SCIENCE, pp. 743-746.

\section{Imaging calcium and signal transduction}

- T.J. Collins et al (April 2, 2002). Mitochondria are morphologically and functionally heterogeneous within cells. EMBO J., pp. 1616-1627.

- T.J. Collins and M.D. Bootman (June 2003). Mitochondria are morphologically heterogeneous within cells. J. EXP. BIOL., pp. 1993-2000

- J. Bruton et al (Aug. 15, 2003). Mitochondrial and myoplasmic $\left[\mathrm{Ca}^{2+}\right]$ in single fibres from mouse limb muscles during repeated tetanic contractions. J. PHYSIOL., pp. 179-190.

- J.D. Bruton et al (Oct. 15, 2003). Mitochondrial function in intact skeletal muscle fibres of creatine kinase deficient mice. J. PHYSIOL., pp. 393-402.

\section{Dental imaging}

- R.A. Couture and C. Hildebolt (April 2000). Quantitative dental radiography with a new photostimulable phosphor system. ORAL SURG. ORAL MED. ORAL PATHOL. ORAL RADIOL. ENDOD., pp. 498-508.

- R.A. Couture and C.F. Hildebolt (January 2002). Precise image-receptor calibration and monitoring of beam quality with a step wedge. DENTOMAXILLOFAC. RADIOL., pp. 5662.

- U. Meyer et al (April 2002). First experience with a public domain computer-aided surgical system. BR. J. ORAL MAXILLOFAC. SURG., pp. 96-104.

\section{Tumor differentiation and soft-tissue motion measurement}

- M.D. Abràmoff et al (October 2000). MRI dynamic color mapping: a new quantitative technique for imaging soft tissue motion in the orbit. INVEST. OPHTHALMOL. VIS. SCI., pp. 3256-3260.

- M.D. Abràmoff, W.J. Niessen and M.A. Viergever (October 2000). Objective quantification of the motion of soft tissues in the orbit. IEEE TRANS. MED. IMAGING, pp. 986-995.

- M.D. Abràmoff et al (September 2001). Patients with persistent pain after enucleation studied by MRI dynamic color mapping and histopathology. INVEST. OPHTHALMOL. VIS. SCI., pp. 2188-2192.

- M.D. Abràmoff and M.A. Viergever (April 2002). Computation and visualization of three-dimensional soft tissue motion in the orbit. IEEE TRANS. MED. IMAGING, pp. 296-304.

\section{Retinal image analysis}

- J. Staal et al (April 2004). Ridge-based vessel segmentation in color images of the retina. IEEE TRANS. MED. IMAGING, pp. 501-509.

- M.D. Abràmoff et al (2004). A spatial truncation approach to the analysis of optical imaging of the retina in humans and cats. Proc. IEEE International Symposium on Biomedical Imaging 2004 2, pp. 1115-1118.

\section{Brain and fat tissue imaging}

- B. Haelewyn et al (September 2003). Desflurane affords greater protection than halothane against focal cerebral ischaemia in the rat. BR. J. ANAESTH., pp. 390-396.

- B. Haelewyn et al (April 2004). Cardioprotective effects of desflurane: effect of timing and duration of administration in rat myocardium. BR. J. ANAESTH., pp. 552-557.

- J.P. Sacha et al (May 2003). Quantification of regional fat volume in rat MRI. Proc. SPIE Medical Imaging, pp. 289-297.

\section{Neuroscience}

- G. Aakalu et al (May 2001). Dynamic visualization of local protein synthesis in hippocampal neurons. NEURON, pp. 489502.

- S.J. Tang et al (Nov. 8, 2001). A role for a rat homolog of staufen in the transport of RNA to neuronal dendrites. NEURON, pp. 463-475.

- M. Zonta et al (2003). Neuron-to-astrocyte signaling is central to the dynamic control of brain microcirculation. NAT. NEUROSCI., pp. 43-50.

\section{Craniofacial surgery simulation}

- T. Stamm et al (December 2003). Validity of a three-dimensional public-domain system for contemporary endodontic research. J. ENDOD., pp. 801-805.

\section{Simulation of cell growth patterns}

- G. Landini and P.M. Iannaccone (April 2000). Modeling of mosaic patterns in chimeric liver and adrenal cortex: algorithmic organogenesis? FASEB J., pp. 823-827.

\section{Telediagnosis and image servers}

- J.D. Parker and J.W. Wallis (October 2003). Systems for Remote Interpretation of Emergency Studies. SEMINARS IN NUCLEAR MEDICINE XXXIII, pp. 324-330.

- M.D. Abràmoff (2003). Impact of Security on Data Transfer in Teleophthalmology for Diabetic Retinopathy Screening over the Internet. Proc. Computer Aided Fundus Imaging and Analysis (CAFIA). 